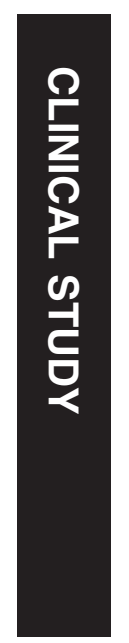

\title{
Remove, rotate, and reimplant: a novel technique for the management of exposed porous anophthalmic implants in eviscerated patients
}

${ }^{1}$ Department of Ophthalmic Plastic and Reconstructive Surgery, Istanbul Beyoglu Eye Research and Training Hospital, Galata, Istanbul, Turkey

\section{Department of} Ophthalmology, Istanbul Beyoglu Eye Research and Training Hospital, Galata, Istanbul, Turkey

${ }^{3}$ Medeniyet University Faculty of Medicine, Department of Ophthalmology, Istanbul, Turkey

Correspondence: P Kaynak, Department of Ophthalmic Plastic and Reconstructive Surgery, Beyoglu Eye Research and Training Hospital, Bereketzade, Beyoglu District, Istanbul 34688, Turkey

Tel: +90 5323668738;

Fax: +90 2122916245

E-mail: pkaynak@gmail.com

Received: 24 May 2013 Accepted in revised form: 12 December 2013 Published online:

7 February 2014

This study had been presented in part in 41st ASOPRS Fall Symposium 2011, Orlando, FL, USA

\begin{abstract}
Purpose To describe and to evaluate a new and relatively easy technique for porous implant exposure repair.

Methods Eleven patients with exposed porous orbital implants after evisceration were included in this study. Five patients with large exposures (diameter $>7 \mathrm{~mm}$ ) and six patients with small exposures of orbital implants (diameter $<7 \mathrm{~mm}$ ) that persisted despite posterior vaulting of the prosthesis and usage of antibiotics and steroids for more than 6 weeks, underwent revision surgery with the remove-rotate-reimplant technique ( $3 R$ technique). Negative microbiological culture taken from the exposed socket surface before surgery was the major inclusion criterion. Five patients with insufficient conjunctival tissue also underwent additional mucosa or hard palate grafting of the defect in addition to the remove-rotate-reimplant procedure.

Results Patients have been followed up for more than 18 months (ranging from 18-30 months). None of them received motility peg insertion after repair. Implant reexposure was detected in one patient during the follow-up period, which was managed by dermis fat grafting with implant removal.

Conclusion The remove-rotate-reimplant technique is an effective surgical method for repairing exposed porous anophthalmic implants after evisceration with a $90 \%$ success in this study. It avoids the removal of the implant from the sclera, which is a traumatic procedure that may lead to the tearing and loss of scleral tissue covering the
\end{abstract}

P Kaynak', GO Karabulut ${ }^{1}$, C Ozturker ${ }^{1}$, I Perente 2 , B Gökyiğit², A Demirok ${ }^{2,3}$ and OF Yilmaz ${ }^{2}$ implant. Saving the porous implant and scleral cover reduces the surgical time and cost.

Eye (2014) 28, 546-552; doi:10.1038/eye.2014.2; published online 7 February 2014

Keywords: porous implant exposure; exposed hydroxyapatite implants; implant exposure repair

\section{Introduction}

Integrated porous orbital implants following evisceration, enucleation, and secondary implantation have become popular to restore volume in anopthalmic sockets. ${ }^{1,2}$ They also permit drilling and peg insertion, and allow better prosthetic retention, movement, and cosmesis. ${ }^{3,4}$

An extensive porous system, permitting fibrovascular ingrowth from adjacent orbital tissues, is thought to decrease the risk of implant extrusion and infection. 3,5 Paradoxically, implant exposure is the most frequent complication of porous implants reported in many series. ${ }^{2,5-8}$ The incidence of porous implant exposure in many studies varies from $0-34 \%$ with varying follow-up durations. ${ }^{2,4,6,8-14}$ Exposure rates have been shown to increase after motility peg insertion in some studies. $2,7,8,12,15$

Surgical techniques for exposure repair are primary revision ${ }^{11}$ and secondary patch grafting using various materials with and without implant removal and exchange. Endogeneous and exogeneous materials have been used for 
grafting. ${ }^{3,4,9,16-20}$ Porous implant exposure management, after evisceration, may necessitate removal of the porous implant from the scleral tissue that requires blunt and sharp dissection of the fibrovascular attachments, which is often a traumatic procedure for the orbital contents and the implant covering and is generally considered a difficult surgical task.

The removed implant is exchanged with a new implant, which is then placed into the remaining sclera and the Tenon's capsule. Conjunctiva is closed primarily or grafts are used to compensate conjunctival shortage, if there is any, to accommodate a proper-sized artificial eye. These techniques require new implant placement and/or coverage, which increases surgical time and cost.

In this study, a new and relatively easy technique used for post-evisceration porous implant exposure repair, in which the exposed implant is reimplanted within the patient's scleral tissue, is described and discussed.

\section{Materials and methods}

A total of 11 patients (six female/five male) who presented with exposed porous orbital implants to the outpatient clinic of Ophthalmic Plastic Surgery Division were included in this study. Mean age of the patients was $37-45$ years. All patients had undergone primary evisceration with posterior expansion sclerotomies and orbital implant insertion during which six of them eventually underwent pegging with titanium sleeve and pegs (Figure 1). Indications for evisceration were globe phthisis due to trauma in six patients, disfigured buphthalmos due to congenital glaucoma in two patients, and disfigured blind eyes in the other three patients after multiple failed vitreoretinal surgeries for proliferative vitreoretinopathy as listed in Table 1.

The size of conjunctival exposure area ranged from $5 \times 5 \mathrm{~mm}$ to $8 \times 10 \mathrm{~mm}$. The scleral exposure sites were larger than the conjunctival exposure sites in all cases ranging between $7 \times 8 \mathrm{~mm}$ to $15 \times 20 \mathrm{~mm}$ (Table 1 ).

The type of implant used in the first operation was a bioceramic implant in six patients, coralline hydroxyapatite in four patients, and porous polyethylene in one patient. Six patients underwent evisceration surgery with expansion sclerotomies and optic neurotomy in our clinic, whereas the other five patients were eviscerated elsewhere.

Implant sizes were either 20 (seven patients) or $22 \mathrm{~mm}$ (four patients). Duration between implantation and occurrence of exposure ranged from 3 years to 6 years. In addition, one patient (case 4) underwent granuloma excision 7 months after the first operation. Four years later, the patient presented with pyogenic granuloma and exposure.

In all, 11 patients with exposures that persisted despite posterior vaulting of the prostheses and usage of antibiotics according to antibiogram for more than 8 weeks, microbial samples were swabbed from exposure site and anophthalmic socket, and sent for culture. The ones with negative results were included in the study and underwent revision surgery via the remove-rotatereimplant (3R) technique under general anesthesia, as described below.

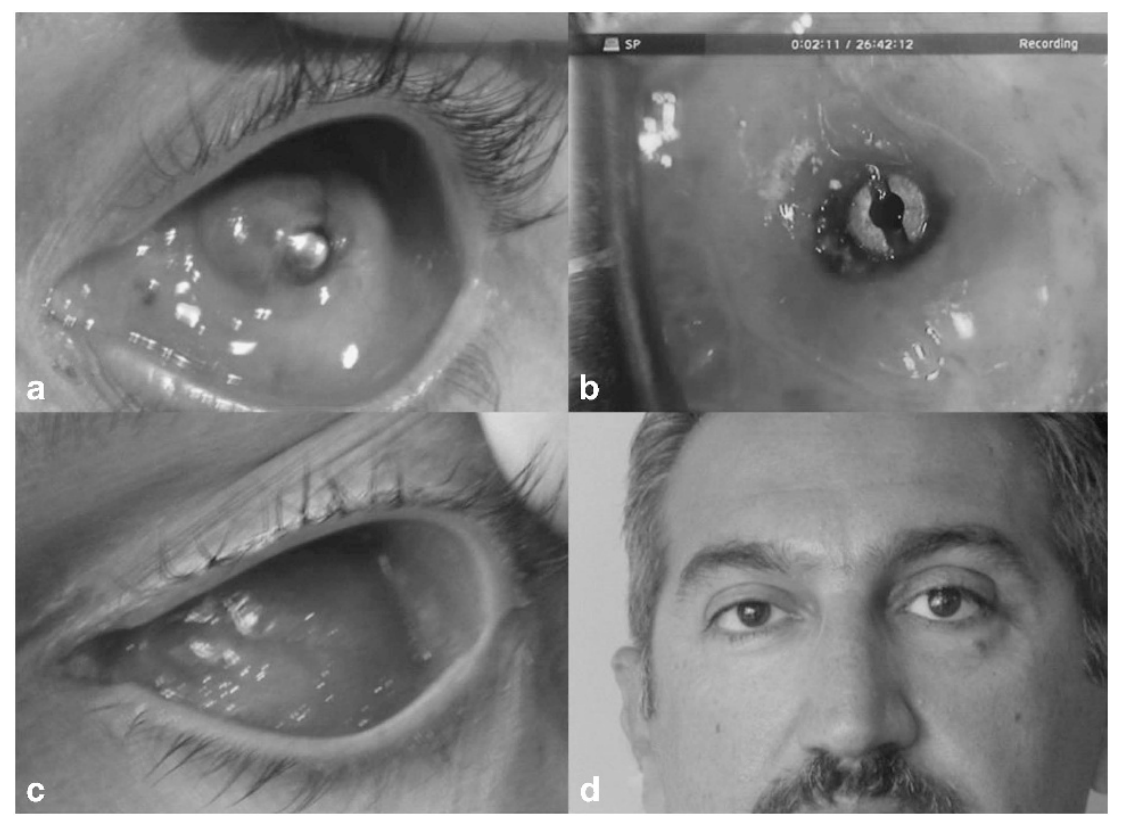

Figure 1 Case 7. Peg surrounded by pyogenic granuloma (a); implant exposure seen after removal of pyogenic granuloma and peg (b); postoperative view (c); patient with prosthetic eye (d). 
Table 1 Summary of cases with implant exposure who were repaired by $3 \mathrm{R}$ technique

\begin{tabular}{lllcccc}
\hline Case (age, sex) & Primary diagnosis & Type of primary implant & $\begin{array}{c}\text { Size of } \\
\text { primary } \\
\text { implant }(m m)\end{array}$ & $\begin{array}{c}\text { Time to } \\
\text { exposure } \\
\text { from first } \\
\text { operation (years) }\end{array}$ & $\begin{array}{c}\text { Size of } \\
\text { defect } \\
\text { (mm } \times \text { mm) }\end{array}$ & $\begin{array}{c}\text { Total } \\
\text { follow-up } \\
\text { time }(\text { months }\end{array}$ \\
\hline $1(18, \mathrm{~F})$ & Trauma, phthisic globe & Bioceramic & 20 & 4 & $5 \times 5$ & 24 \\
$2(55, \mathrm{M})^{\mathrm{a}, \mathrm{b}}$ & Trauma, phthisic globe & Coralline hydroxyapatite & 20 & 3 & $7 \times 10$ & 30 \\
$3(34, \mathrm{~F})^{\mathrm{a}, \mathrm{c}}$ & Trauma, phthisic globe & Bioceramic & 22 & 5 & $8 \times 8$ & 24 \\
$4(18, \mathrm{~F})$ & Congenital glaucoma & Bioceramic & 20 & 5.5 & $6 \times 5$ & 28 \\
$5(42, \mathrm{M})^{\mathrm{a}, \mathrm{b}}$ & Retinal detachment and PVR & Coralline hydroxyapatite & 22 & 3 & $8 \times 10$ & 28 \\
$6(50, \mathrm{~F})$ & Trauma, phthisic globe & Bioceramic & 20 & 6 & $5 \times 5$ & 32 \\
$7(47, \mathrm{M})^{\mathrm{a}}$ & Trauma, phthisic globe & Bioceramic & 22 & 5 & $6 \times 5$ & 27 \\
$8(33, \mathrm{~F})^{\mathrm{a}}$ & Congenital glaucoma & Coralline hydroxyapatite & 20 & 6 & $5 \times 7$ & 34 \\
$9(48 ; \mathrm{M})^{\mathrm{b}}$ & Retinal detachment and PVR & Coralline hydroxyapatite & 22 & 4 & $9 \times 9$ & 26 \\
$10(32 ; \mathrm{F})^{\mathrm{a}, \mathrm{b}}$ & Trauma, phthisic globe & Porous polyethylene & 20 & 4 & $7 \times 8$ & 36 \\
$11(41 ; \mathrm{M})$ & Retinal detachment and PVR & Bioceramic & 20 & 6 & $7 \times 6$ & 35 \\
\hline
\end{tabular}

a Pegged implants.

${ }^{\mathrm{b}}$ Oral mucosa graft needed for conjunctival repair.

${ }^{\mathrm{c}}$ Hard palate graft needed for conjunctival repair.

\section{Surgical technique}

Conjunctiva was incised at the edge of the defect extending medially and laterally, after subconjunctival and retrobulber injections of $2-4$ cc. $0.3 \%$ bupivacaine hydrochloride and $2 \%$ lidocaine hydrochloride with $1: 125000$ epinephrine hydrochloride mixture for hemostasis and postoperative pain relief. Previously implanted pegs and sleeves were removed. The conjunctiva and the Tenon's fascia were undermined and the inward rolled epithelial rim was removed circumferentially over the exposed implant. Scleral edges over the exposure were reached. Six extraocular muscles were identified, tagged with $6 / 0$ polyglactin sutures, and then disinserted from sclera. The porous implant was removed (explanted) within scleral leaves of the primary expanded evisceration and soaked in a $60 \mathrm{ml} \mathrm{1:1} \mathrm{mixture}$ of gentamicin $40 \mathrm{mg} / \mathrm{ml}$ and cephuroxime $500 \mathrm{mg} / \mathrm{ml}$, then it was rotated and reimplanted in the posterior Tenon's fascia bag, with the largest sclera-covered side facing anteriorly. Four rectangular scleral windows were excised at the premarked appropriate sites for reinsertion of muscles. The extraocular muscles were reattached to the sclera at these scleral openings to enable further fibrovascularization of the implant (Figure 2).

In six patients with small exposures, Tenon's fascia and conjunctiva were closed under least tension with running absorbable $6 / 0$ polyglactin sutures when an adequatesized conformer was possible in the anophthalmic socket. Four of five patients, with large exposures of orbital implants ( $>7 \mathrm{~mm}$ ), did not have sufficient conjunctival tissue and additional buccal mucosal graftings were performed on them. One patient, who had undergone three buccal mucosa grafting surgeries for exposure repairs previously underwent hard palate grafting of the defect, due to scarring of buccal mucosal donor site, in addition to the remove-rotate-reimplant procedure (Figure 3).

Postoperative antibiotic ointment, a large socket conformer, and sterile dressing were placed in the socket and the socket was patched under moderate tension for 3 days. Systemic and topical antibiotics were prescribed postoperatively. All patients were examined on days 3 and 15 after surgery, and then every 3 weeks. The prosthetic fittings were done between $4-8$ weeks after surgery. The patients were examined every 3 months after prosthetic fitting.

\section{Results}

All patients underwent uneventful surgery, in which the primary porous implants were removed within the scleral remnants, rotated, and reimplanted into the posterior Tenon's bag in contact with the orbital fat. The posterior and anterior Tenon's layers and the conjunctiva were separately closed in front of the implant and the anophthalmic socket surface defect repaired by buccal mucosa in four patients and hard palate grafting in one patient, when mucosal defect was large.

Patients were followed up for more than 24 months (24-36 months). No patient underwent repegging after $3 \mathrm{R}$ repair of exposure. One patient (case 2) experienced mild infection with purulent discharge postoperatively, which responded well to topical antibiotic ointment treatment. Implant reexposure was detected in this patient 7 months later and the patient underwent dermis fat grafting with removal of implant. No severe infection was observed in any of the other patients during the follow-up period. 


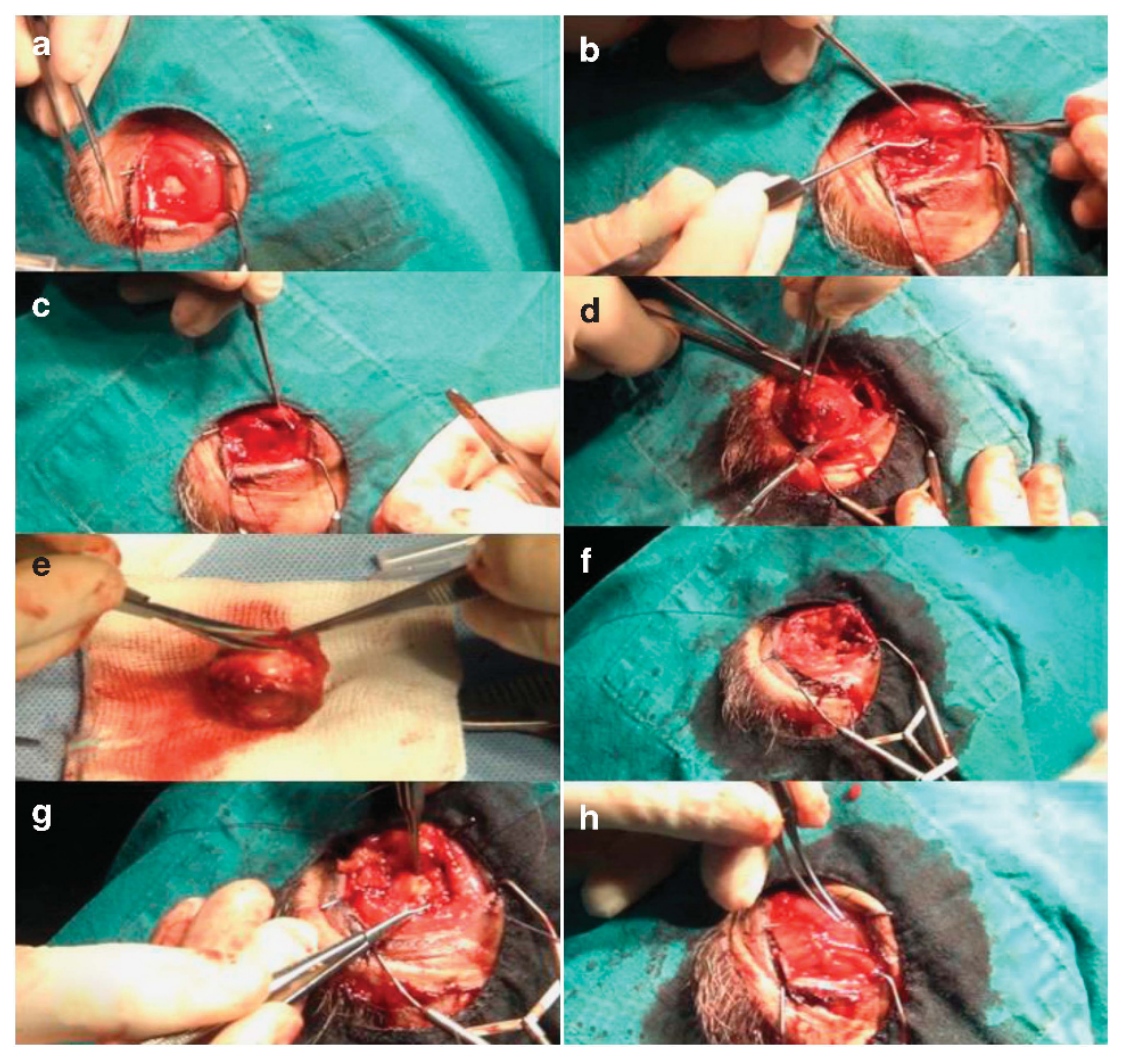

Figure 2 Surgical technique for implant revision: preoperative view (a); undermined conjunctiva and Tenon's fascia edges extending medially and laterally (b); identified extraocular muscles tagged with 6/0 polyglactin sutures disinserted from sclera (c); explantation of porous implant (d); explanted porous implant within scleral remnants (e); Tenon's capsule bag after removal of implant (f); rotation and reimplantation of implant with the largest sclera-covered side facing anteriorly, reattachment of muscles to scleral windows (g); closure of Tenon's fascia and conjunctiva with running sutures (h).

Fornices were deep enough in all patients to accommodate the ocular prosthetic eyes. All patients reported that they were satisfied with their cosmesis.

\section{Discussion}

Although porous systems with fibrovascularized ingrowths are thought to decrease the risk of implant extrusion, conjunctival dehiscence and implant exposure are still important complications of porous implants. ${ }^{2,5-8}$ These complications are managed by surgical procedures that usually necessitate removal of the porous implant, a new implant placement, and meticulous repair of the remaining soft tissues of the anopthalmic socket. Porous implant removal, particularly after evisceration is difficult because of the 'velcro' effect in between the irregular surface of the porous implant and the sclera. This surgical intervention almost always damages the sclera covering the implant and orbital soft tissues. Secondary wrapping of the new porous implant requires autologous or alloplastic grafts such as banked sclera, bovine pericardium ${ }^{4}$ or dura, ${ }^{6}$ which increase surgical expenses, in addition to the wasted porous implant.

Causes of implant exposure are infection, edema, hemorrhage, and exposure and are related to wrapping material, pegging, surgical technique used, too-large implant size, and surgeon's experience as reported by Viswanathan et al. ${ }^{4}$ Tension on wound, spicules on HAP implant surface, ${ }^{21}$ pressure of prosthesis on conjunctival surface, ${ }^{9}$ inadequate vascularization of implant, ${ }^{2}$ tissue restitution, or long-term adductive rotation of the implant $^{22}$ are other factors that cause anterior tissue breakdown. Shields et $a l^{2}$ suggested that conjunctival defects that occurred several months or later, might be due to a poorly fitting prostheses causing pressure necrosis, anterior malplacement of the implant, inadequate closure of the Tenon's fascia over the implant, or lack of implant vascularization. ${ }^{2}$ All of our patients had prostheses with proper fitting and had late exposures, suggesting that the anterior malplacement of the porous implant caused posterior dragging of Tenon's capsule. Sagoo and Rose $^{22}$ termed the anterior migration of implant because of tissue restitution during healing as 


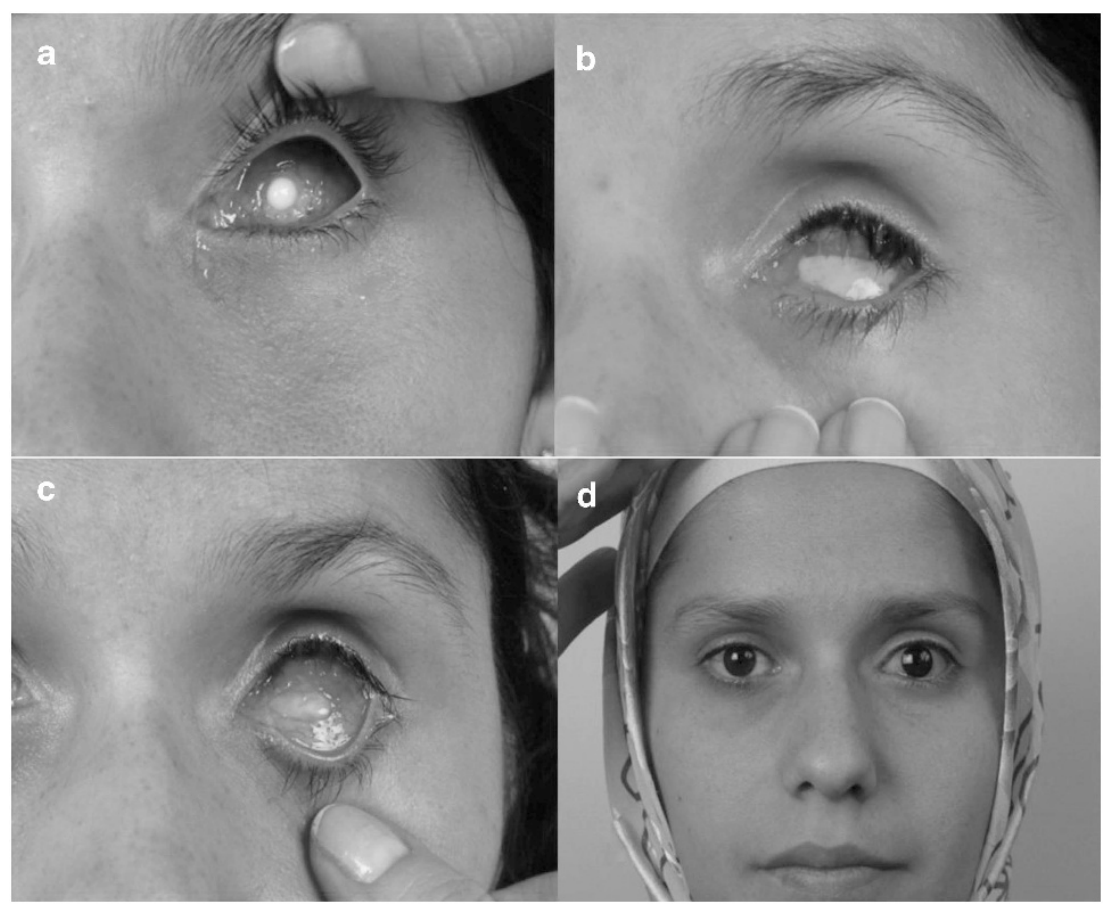

Figure 3 Case 3. Orbital implant exposure with peg (a); postoperative view, conjunctival defect repaired with hard palate graft (b); late postoperative view with conjunctivalized hard palate (c); patient with prosthetic eye (d).

the 'cactus syndrome' and described the syndrome as when an orbital implant was forced into the tissues during implantation and the implant surface dragged the superficial tissues into the depths of the socket. The 3R technique enables easy insertion of the sclera-covered implant into the posterior Tenon's fascia bag.

Spontaneous healing of porous implant exposure was noted in $15.8 \%$ of HAP implants. ${ }^{6}$ Pelletier et al ${ }^{16}$ recommended repairing of the defect if it does not close within 8 weeks of diagnosis. Initial management of exposure included posterior vaulting of the prosthetic eye and administration of antibiotic ointment and drops for 8 weeks in our study, as recommended in some reports. ${ }^{12,16,17}$

We performed the operation in case of a persisting or enlarging defect despite removal of the artificial eye and placement of an adequate-sized and vaulted conformer with smooth edges and use of topical antibiotics effective on the cultured microorganisms taken from the anophthalmic socket surface according to the antibiogram.

We only reimplanted the exposed porous implants with negative culture results taken secondarily before surgery to prevent the possible spread of infection. In the study of Toft et $a l^{23} 87 \%$ of removed implants showed inflammation to various extents, possibly having undetected microorganisms within the implant. They routinely soaked the new implant in gentamicin, and gave one dose of cefuroxime intravenously at the time of operation and oral antibiotics in the postoperative period without reported infections. Jordan et $a l^{24}$ found microorganisms in $33 \%$ and Chuo et $a l^{25}$ in $67 \%$ of explanted implants. Implant infection may occur late with or even without pegging. ${ }^{15}$ Generally, antibiotics used intraoperatively and postoperatively have been proven to avoid infection. As an additional caution to prevent a possible infection, we impregnated the implant in a mixture of gentamicin and cefuroxime just before implantation. No postoperative infection was seen in our study group except one patient who had secretion and edema postoperatively, suggesting low-grade infection, which responded well to topical and systemic cephalosporin administration. This patient had a history of previous multiple exposures of orbital implant and a large defect closed with $3 \mathrm{R}$ technique combined with oral mucosa grafting. The patient presented with reexposure 7 months later and underwent dermis fat grafting with removal of implant. The other patients who received oral mucosa graftings did not show any signs of infection.

Sclera-covered part of the implant, which we have rotated anteriorly, also serves as a perfect lining to protect the free buccal mucosa or hard palate graft from the spicules of the porous material that may accelerate the breakdown of graft tissue. ${ }^{21}$ The need for exogenous graft material such as banked sclera for scleral defect closure is avoided by this method. Liao et $a l^{3}$ have 
reported banked scleral graft melting over the porous material. We never used autologous or exogenous grafts over exposed porous implants followed by primary closure of the conjunctiva over the graft, but we preferred to graft the larger mucosal defects to overcome wound tension of primary closure, which is a well-known reason of anophthalmic implant exposure. ${ }^{21}$ Better conjunctival fornices are formed by adding this in our surgical course to accommodate an adequate-sized prosthetic eye.

Madill and Maclean ${ }^{26}$ described a similar technique as a primary procedure in patients listed for traditional evisceration in which they eviscerated the contents of the globe after enucleation to use as a scleral shell to wrap the implant and they attached the muscles onto the scleral covering. The corneal opening of the scleral shell was rotated posteriorly and replaced in the orbit in this technique. However, our technique was performed only in patients with exposed porous implants and both the previous implant and the scleral covering were reused to avoid extended surgical manipulation, duration, and cost. The remove-rotate-reimplant (3R) technique described in our study saves the implant within the patient's own scleral tissue too. Removal of the implant within scleral remnants covering the irregular surface of the implant is easier and less traumatic to the sclera when compared with removal of the implant from the scleral tissue. Exposed porous implant, partially wrapped with patients own scleral tissue facing anteriorly is reimplanted easily into the posterior Tenon's capsule. It avoids new implant and autologous or alloplastic graft usage. The surgical time is generally shorter.

Implant exposure results in loss of tissue and a decrease in conjunctival area. ${ }^{9}$ Kim et al ${ }^{21}$ recommended repair with a patch graft to increase the conjunctival area. Management of porous sphere implant exposure includes two main graft techniques: those with exogenous material versus those with autogenous material. Risk of immunological reaction and infection is seen less in autogenous graft materials such as temporalis fascia, ${ }^{4,16,17}$ dermis fat grafts, ${ }^{9}$ retroauricular myoperiosteal graft, ${ }^{3}$ frontal periosteal graft, ${ }^{18}$ Müller muscle flap, ${ }^{19}$ and implant capsule. ${ }^{20}$ Exogenous scleral patch grafts carry the risk of transmission of slow virus infections. ${ }^{27,28}$ Liao et $a l^{3}$ concluded that banked sclera may melt if vascular supply was insufficient and it might contain infectious agents. Also as a homologous material, it may generate more inflammation than autologous tissue. In dermis fat grafting technique, they eliminated the implant exposure by burying it down. ${ }^{9}$ Tawfik et al ${ }^{20}$ described another technique using implant capsule for closure of exposure, which occurred in an enucleated socket. In addition, their technique creates a too-thin flap and injury to the extraocular muscles, which results in incomplete closure of defect. Our technique is suitable for repairing exposures after eviscerations. Among five patients with large exposures who underwent the remove-rotate-reimplant operation, four patients received additional oral mucosa grafting and one patient needed hard palate grafting for conjunctival defect closure. Hard palate grafting was done in a patient with history of multiple implant exposures, because oral mucosa donor sites were scarred due to previous grafts taken for anophthalmic socket reconstruction.

Combined explant-implant procedure in the same surgical intervention described by Toft et $a l^{23}$ saved the patient from unsuccessful attempts to close the conjunctiva and from a second operation. In their technique, they removed the implant and replaced it with a new implant in the same procedure. Our technique eliminated multiple operations and provided cost effectiveness by inserting the same porous implant.

In conclusion, the 'remove-rotate-reimplant (3R)' technique minimizes the difficulty of detaching the porous implants from the scleral petals and socket contents. Considering the relatively short follow-up time (24-36 months) in our study group, 3R technique is effective in repairing exposed porous anophthalmic implants in eviscerated patients, saving patients from multiple operations and providing cost effectiveness.

\section{Summary}

\section{What was known before}

- Porous implant exposure management after evisceration may necessitate removal of the porous implant from the scleral tissue, which requires blunt and sharp dissection of the fibrovascular attachments, which is often a traumatic procedure for the orbital contents and the implant covering and is generally considered a difficult surgical task.

What this study adds

- A new and relatively easy technique used for postevisceration porous implant exposure repair, in which the exposed implant is reimplanted within the patient's scleral tissue, is described and discussed.

\section{Conflict of interest}

The authors declare no conflict of interest.

\section{References}

1 Perry AC. Integrated orbital implants. Adv Ophthalmic Plast Reconstr Surg 1990; 8: 75-81.

2 Shields CL, Shields JA, Potter PD, Singh AD. Problems with the hydroxyapatite orbital implant: experience with 250 consecutive cases. Br J Ophthalmol 1994; 78: 702-706. 
3 Liao SL, Kao SCS, Tseng JHS, Lin LL-K. Surgical coverage of exposed hydroxyapatite implant with retroauricular myoperiosteal graft. Br J Ophthalmol 2005; 89: 92-95.

4 Viswanathan P, Sagoo MS, Olver JM. UK national survey of enucleation, evisceration and orbital implant trends. Br J Ophthalmol 2007; 91: 616-619.

5 Shields CL, Shields JA, Eagle Jr RC, De Potter P. Histopathologic evidence of fibrovascular ingrowth four weeks after placement of the hydroxyapatite orbital implant. Am J Ophthalmol 1991; 111: 363-366.

6 Custer PL, Trinkaus KM. Porous implant exposure: incidence, management, and morbidity. Ophthal Plast Reconstr Surg 2007; 23(1): 1-7.

7 Oestreicher JH, Liu E, Berkowitz M. Complications of hydroxyapatite orbital implants. A review of 100 consecutive cases and a comparison of dexon mesh (polyglycolic acid) with scleral wrapping. Ophthalmology 1997; 104: 324-329.

8 Nunnery WR, Heinz GW, Bonnin JM, Martin RT, Cepela MA. Exposure rate of hydroxyapatite spheres in the anophthalmic socket: histopathologic correlation and composition with silicone sphere implants. Ophthal Plast Reconstr Surg 1993; 9: 96-104.

9 Buettner H, Bartley GB. Tissue breakdown and exposure associated with orbital hydroxyapatite implants. Am J Ophthalmol 1992; 113: 669-673.

10 Christmas NJ, Gordon CD, Murray TG, Tse D, Johnson T, Garonzik $\mathrm{S}$ et al. Intraorbital implants after enucleation and their complications: a 10-year review. Arch Ophthalmol 1998; 116: 1199-1203.

11 Remulla HD, Rubin PA, Shore JW, Sutula FC, Townsend DJ, Woog JJ et al. Complications of porous spherical implants. Ophthalmology 1995; 102: 586-593.

12 Jordan DR. Problems after evisceration surgery with porous orbital implants: experience with 86 patients. Ophthal Plast Reconstr Surg 2004; 20(5): 374-380.

13 Dutton JJ. Coralline hydroxyapatite as an ocular implant. Ophthalmology 1991; 98: 370-377.

14 Suter AJ, Molteno CB, Bevin TH, Fulton JD, Herbison P. Long-term follow-up of bone derived hydroxyapatite orbital implants. Br J Ophthalmol 2002; 86: 1287-1292.

15 Karsloglu S, Serin D, Simsek I, Ziylan S. Implant infection in porous orbital implants. Ophthal Plast Reconstr Surg 2006; 22(6): 461-466.
16 Pelletier CR, Jordan DR, Gilberg SM. Use of temporalis fascia for exposed hydroxyapatite. Ophthal Plast Reconstr Surg 1998; 14: 198-203.

17 Sagoo MS, Olver JM. Autogenous temporalis fascia patch graft for porous polyethylene (Medpor) sphere orbital implant exposure. Br J Ophthalmol 2004; 88: 942-946.

18 Massry GG, Holds JB. Frontal periosteum as an exposed orbital implant cover. Ophthal Plast Reconstr Surg 1999; 15: 79-82.

19 Rosen CE. The Müller muscle flap for repair of an exposed hydroxyapatite orbital implant. Ophthal Plast Reconstr Surg 1998; 14(3): 204-207.

20 Tawfik HA, Budin H, Dutton JJ. Repair of exposed porous polyethylene implants utilizing flaps from the implant capsule. Ophthalmology 2005; 112: 516-523.

21 Kim TD, Goldberg RA, Shorr N, Steinsapar KD. Management of exposed hydroxyapatite orbital implants. Ophthalmology 1994; 101: 1709-1715.

22 Sagoo MS, Rose GE. Mechanisms and treatment of extruding intraconal implants, socket aging and tissue restitution (the 'cactus syndrome'). Arch Ophthalmol 2007; 125(12): 1616-1620.

23 Toft PB, Roed Rasmussen ML, Prause JU. One-stage explant-implant procedure of exposed porous orbital implants. Acta Ophthalmol 2012; 90: 210-214.

24 Jordan DR, Brownstein S, Faraji H. Clinicopathologic analysis of 15 explanted hydroxyapatite implants. Opthal Plast Reconstr Surg 2004; 20: 285-290.

25 Chuo JY, Dolman PJ, Ng TL, Buffam FV, White VA. Clinical and histopathologic review of 18 explanted porous polyethylene orbital implants. Ophthalmology 2009; 116: 349-354.

26 Madill SA, Maclean H. Enucleation with reverse replacement of sclera as an alternative to conventional evisceration. Orbit 2005; 24: 23-28.

27 Fountain JA, Helveston EM. A long term follow-up study of sclera grafting for exposed or extruded orbital implants. Am J Ophthalmol 1982; 93: 52-56.

28 Goldberg MF. A simplified sclera graft technique for covering an exposed orbital implant. Ophthalmic Surg 1988; 19: 206-211. 